\title{
Genital mycoplasma and preterm birth: a difficult puzzle to solve. Letter re: Genital mycoplasma infection and spontaneous preterm birth outcome: a prospective cohort study
}

\author{
Andrew Kotaska ${ }^{1}$ and Lesley Paulette ${ }^{2}$ \\ ${ }^{1}$ University of Manitoba Faculty of Medicine \\ ${ }^{2}$ Affiliation not available
}

October 23, 2021

\section{Genital mycoplasma and preterm birth: a difficult puzzle to solve.}

Letter re: Genital Mycoplasma infection and spontaneous preterm birth outcome: a prospective cohort study Sir.

In their recent paper, Cunha and colleagues analyzed data from a diverse group of 1349 Brazilian women who participated in a 2011 study evaluating etiological factors associated with preterm birth. 11Cunha GKP, Bastos LB, de Freitas SF, Cavalli RC, Quintana SM. Genital mycoplasma infection and spontaneous preterm birth outcome: a prospective cohort study. BJOG https://doi-org.uml.idm.oclc.org/10.1111/14710528.16949 Women were interviewed and examined at 20-25 weeks' gestation, and cervicovaginal cultures were taken for mycoplasma, ureaplasma, and bacterial vaginosis. Women were followed until delivery, and clinical associations with preterm birth were evaluated. Positive associations were found for shortened cervix and a history of prior preterm birth. No association was found for infection with mycoplasma, ureaplasma, or bacterial vaginosis. They concluded that "genital mycoplasma infection was not a risk of spontaneous preterm birth, even with other abnormal vaginal microbiota conditions."

There is a flaw in their methodology and conclusion. In the original study, women with a positive culture for ureaplasma or mycoplasma were treated with azithromycin, and those with bacterial vaginosis were treated with oral metronidazole. Treatment would have reduced the presence of these pathogens, limiting their ability to cause preterm birth. Accordingly, this study shows that women with treated mycoplasma, ureaplasma or bacterial vaginosis do not have an increased incidence of preterm birth; however, no comment can be made on whether untreated infection causes preterm birth.

Presumably the original investigators who performed the study treated women with genital infection because they believed it might prevent preterm birth; and there lies the rub. An observational study using vaginal culture results unavailable until after birth has shown an association between untreated mycoplasma infection and preterm birth.22Foxman B, Wen A, Srinivasan U, et al. Mycoplasma, bacterial vaginosis, associated bacteria BVAB3, race, and risk of preterm birth in a high-risk cohort. Am J Obstet Gynecol 2014;210:226.e17. However, in order to determine whether mycoplasma causes preterm birth and whether treatment prevents it, a randomized trial of treatment or placebo in infected women at elevated risk of preterm birth is needed. This may not be easily feasible. Ethically, investigators may be able to demonstrate research equipoise: that we do not yet know whether treatment reduces preterm birth associated with mycoplasma infection. However, women found to have genital mycoplasma who are at increased risk of preterm birth would need to believe that the benefits and harms of treating or not treating are equal. Given the relative safety of antibiotics and the high morbidity of preterm birth, practically, equipoise may be hard to find. 
In Northern Canada, with high rates of sexually transmitted infections including mycoplasma, we suspect a causal relationship with preterm birth and late miscarriage. Based on observational studies and anecdotal evidence, our practice is to screen women with a history of prior preterm birth, late miscarriage, or short cervix for ureaplasma, mycoplasma, and bacterial vaginosis in the second trimester and treat those who are positive. $^{2,33 T a y l o r-R o b i n s o n ~ D, ~ L a m o n t ~ R . ~ M y c o p l a s m a s ~ i n ~ p r e g n a n c y . ~ B J O G ~ 2011 ; 118: 164-174 . ' 44 M o r e n c y ~}$ AM, Bujold E. The Effect of Second-Trimester Antibiotic Therapy on the Rate of Preterm Birth. J Obstet Gynaecol Can 2007;29(1):35-44 We also test women with threatened preterm labour remote from term and treat those who are positive, sometimes with marked reduction in symptoms. In keeping with TaylorRobinson's paper, treatment failures with azithromycin used for Mycoplasma hominis have prompted a switch to Clindamycin, whose activity against both mycoplasma and bacterial vaginosis may explain its better performance than metronidazole in the prevention of preterm birth. ${ }^{3,4}$

We await better evidence, but are not holding our breath. 